\title{
mTOR hyperactivation in Down Syndrome underlies deficits in autophagy induction, autophagosome formation, and mitophagy
}

Matteo Bordi ${ }^{1,2,3}$, Sandipkumar Darji $\mathbb{1}^{1}$, Yutaka Sato ${ }^{1}$, Marian Mellén ${ }^{4}$, Martin J. Berg ${ }^{1}$, Asok Kumar ${ }^{1}$, Ying Jiang ${ }^{1,2}$ and Ralph A. Nixon ${ }^{1,2,5,6}$

\begin{abstract}
Down syndrome (DS), a complex genetic disorder caused by chromosome 21 trisomy, is associated with mitochondrial dysfunction leading to the accumulation of damaged mitochondria. Here we report that mitophagy, a form of selective autophagy activated to clear damaged mitochondria is deficient in primary human fibroblasts derived from individuals with DS leading to accumulation of damaged mitochondria with consequent increases in oxidative stress. We identified two molecular bases for this mitophagy deficiency: PINK1/PARKIN impairment and abnormal suppression of macroautophagy. First, strongly downregulated PARKIN and the mitophagic adaptor protein SQSTM1/p62 delays PINK1 activation to impair mitophagy induction after mitochondrial depolarization by CCCP or antimycin A plus oligomycin. Secondly, mTOR is strongly hyper-activated, which globally suppresses macroautophagy induction and the transcriptional expression of proteins critical for autophagosome formation such as ATG7, ATG3 and FOXO1. Notably, inhibition of mTOR complex 1 (mTORC1) and complex 2 (mTORC2) using AZD8055 (AZD) restores autophagy flux, PARKIN/PINK initiation of mitophagy, and the clearance of damaged mitochondria by mitophagy. These results recommend mTORC1-mTORC2 inhibition as a promising candidate therapeutic strategy for Down Syndrome.
\end{abstract}

\section{Introduction}

Down syndrome (DS, HSA21), among the most common genetic disorders, is caused by a total or partial trisomy of chromosome $21^{1}$. Individuals with DS display a considerable range of symptoms, including neurocognitive disabilities of variable severity, due to the high genetic complexity and adaptive mechanisms that partially offset the presence of an extra copy of HSA $21^{2}$. Additionally, DS patients invariably develop Alzheimer's disease (AD) neuropathobiology by the fifth decade of life $^{3}$ with dementia onset by the age of 65 . AD emergence in DS is

Correspondence: Matteo Bordi (matteobordi@gmail.com)

${ }^{1}$ Center for Dementia Research, Nathan Kline Institute, Orangeburg, NY, USA ${ }^{2}$ Department of Psychiatry, New York University Langone Medical Center, New York, NY, USA

Full list of author information is available at the end of the article.

Edited by G.M. Fimia likely attributable to the aneuploidy of the APP gene although the involvement of other genes localized on $\mathrm{Chr}$ 21, such as RCAN1 and DYRK1A, cannot be excluded ${ }^{4}$. Mitochondrial anomalies produce deficits in DS brain and other tissues, including impaired ATP production, oxidative stress and increased vulnerability to cell death ${ }^{5-9}$. Similar mitochondrial abnormalities develop in mouse models of DS, including Ts1 lacking an extra APP allele ${ }^{10}$.

Studies have shown that mitochondrial damage triggers their rapid elimination by macroautophagy ${ }^{11}$, prompting us to consider whether macroautophagy deficits form a basis for the abnormal mitochondrial phenotype and its pathological consequences. Macroautophagy (hereafter referred to as autophagy) is a major lysosomal degradative pathway for the elimination and recycling of obsolete and damaged cytoplasmic components and turnover of vesicular organelles, including mitochondria ${ }^{11,12}$. Compelling

\section{(c) The Author(s) 2019}

(c) (i) Open Access This article is licensed under a Creative Commons Attribution 4.0 International License, which permits use, sharing, adaptation, distribution and reproduction cc) in any medium or format, as long as you give appropriate credit to the original author(s) and the source, provide a link to the Creative Commons license, and indicate if changes were made. The images or other third party material in this article are included in the article's Creative Commons license, unless indicated otherwise in a credit line to the material. If material is not included in the article's Creative Commons license and your intended use is not permitted by statutory regulation or exceeds the permitted use, you will need to obtain permission directly from the copyright holder. To view a copy of this license, visit http://creativecommons.org/licenses/by/4.0/. 
genetic and pathological evidence has implicated autophagy dysfunction in the pathogenesis of multiple neurodegenerative diseases, including $A D^{13,14}$.

Various forms of selective autophagy have been identified, including the removal of damaged mitochondria (mitophagy $)^{15}$. Mitophagy is initiated by signaling events following mitochondrial membrane depolarization leading to the recruitment of specific adaptors including PINK1-PARKIN representing the most wellcharacterized pathway. Herein mitophagy is activated with PINK1 accumulation on the mitochondrial outer membrane upon mitochondrial depolarization, then phosphorylating ubiquitin promoting translocation of the E3 ubiquitin ligase PARKIN (PARK2) ${ }^{16,17}$. PARKIN builds ubiquitin chains on mitochondrial external membrane proteins recruiting specific autophagic cargo receptors, such as p62 (SQSTM1) and NDP52, facilitating sequestration into the autophagosome (APs) ${ }^{17,18}$. Moving forward, mitophagy and bulk autophagy employ similar sequestration and clearance steps; however, the differential regulation of these two processes is not fully understood.

The mTOR complex1 (mTORC1), a master regulatory protein kinase $^{19}$, negatively regulates autophagy by downregulating the ULK1 complex ${ }^{20}$. mTORC1 itself is positively regulated by the small GTPase Rheb on lysosomes $^{21}$, and by the PI3K/AKT cascade downstream of receptor tyrosine kinases ${ }^{22}$. Inhibiting $\mathrm{mTORC} 1$ with activation of ULK1 complex stimulates downstream components of the autophagic pathway, such as PI3K-cIII complex and ATG-related proteins, resulting in nucleation, elongation and formation of $\mathrm{APs}^{23}$. ATGs -7, -3 , and -5 are essential proteins for the correct formation of APs, are involved in two ubiquitin-like systems and binding of LC3/GABARAPs with phosphatidylethanolamine (PE) ${ }^{24}$. Interestingly, studies of DS frontal cortex and hippocampus report aberrant hyperactivation of the AKT/ mTOR signaling pathway in DS brain ${ }^{25,26}$ suggesting imbalances in autophagy flux regulation in DS leads to negative effects on mitochondrial turnover.

Here, we establish mitophagy impairment in DS fibroblasts and identify two underlying molecular mechanisms. First, abnormally lowered levels of PARKIN coupled to altered PINK1 activation induce mitochondrial depolarization. Secondly, and more significantly in this model is mitophagy suppression linked to down-regulated rate of autophagy caused by mTOR hyper activity and accompanying reduction in expression of key ATG proteins. We further demonstrate that mitophagy and overall autophagy can be rescued by inhibiting mTORC1 and mTORC2 with AZD8055, a novel inhibitor of mTOR kinase activity ${ }^{27}$, suggesting innovative avenues for treatment of DS.

\section{Materials and methods}

\section{Cell culture and transfections}

Human forearm skin fibroblasts from DS and diploid $(2 \mathrm{~N})$ age-matched controls (5 months, 2 and 5 years old) from the Coriell Cell Repositories (AG06922, AG07095, AG07096, GM08680, GM05381, AG04823) were cultured in DMEM medium (Life Technologies \#11995-065) containing $10 \%$ fetal bovine serum (Life Technologies \#10270), $100 \mathrm{mg} / \mathrm{L}$ sodium pyruvate, 100 units $/ \mathrm{mL}$ Penicillin-Streptomycin (Life Technologies \#15140-122) at $37^{\circ} \mathrm{C}$ in a $5 \% \mathrm{CO} 2$ atmosphere. Cell passage number ranged from $\mathrm{p} 7$ to $\mathrm{p} 12$, and cells at $85-90 \%$ confluency were used throughout the study. Fibroblasts were seeded on glass coverslips in 12-well dishes for immunolabeling or $100-150 \mathrm{~mm}$ dishes for biochemical applications as follows.

\section{Cell treatment}

As lysosomal-inhibiting agents, we used the following: (a) $10 \mu \mathrm{M}$ Leupeptin inhibitor of cysteinyl and serine proteases) and Pepstatin A (aspartyl proteases)(Peptide Institute, Japan) for $24 \mathrm{~h}$; (b) $10 \mathrm{nM}$ Bafilomycin A (Bafil), a specific inhibitor of the lysosomal vacuolar type $\mathrm{H}^{+}$ATPase (V-ATPase) that blocks lysosomal degradation ${ }^{28}$ (Sigma B1793) for $6 \mathrm{~h}$; (c) $50 \mathrm{nM}$ Concanamycin A (ConA), a second potent V-ATPase inhibitor ${ }^{29}$ (Sigma 27689) for $2 \mathrm{~h}$. For mitochondria depletion via induced mitochondrial membrane depolarization, cells were treated with $20 \mu \mathrm{M} \mathrm{CCCP}^{15}$ (Sigma C2759) for $6 \mathrm{~h}$ or with a combination of $1 \mu \mathrm{M}$ antimycin $\mathrm{A}$ and $1 \mu \mathrm{M}$ oligomycin (AA/OA) inhibiting the electron transport chain complex III and of ATP synthase, respectively ${ }^{28}$ (Sigma A8674/ O4876) for 1, 3, 6 and 24h. Cells were treated with $0.1 \mu \mathrm{M}$ AZD8055 (AZD), a recently introduced ATP-competitive inhibitor directly targeting the mTOR catalytic site, blocking both mTORC1 and mTORC2 ${ }^{30}$ (Selleckchem) for 2,4 and $8 \mathrm{~h}$ as reported in figure legends.

\section{RNA sequencing (RNA-Seq)}

RNA was extracted from human fibroblasts using RNeasy Micro Kit (Qiagen) according to the manufacturer's specifications and digested with DNAse I (Sigma). RNA quantity and quality were determined with a Nanodrop 1000 spectrophotometer (Wilmington, DE) and Agilent 2100 Bioanalyzer system. Three biological replicas were produced per each cell line. $500 \mathrm{ng}$ of total RNA were prepared using TruSeq RNA Sample prep kit V2 (Ilumina Inc., San Diego, CA, USA) following manufacturers' instructions for further sequencing in HiSeq 2000 (Ilumina Inc.). Quality of libraries was assessed using an Agilent 2100 Bioanalyzer system. We obtained on average 52 million $50 \mathrm{bp}$ single-end reads and $50 \mathrm{bp}$ paired-end reads per sample that were separately aligned 
to the human genome (hg19, GRCh37) downloaded from University of California Santa Cruz Genomics Institute. STAR software (version 2.3.1z) was used for processing reads with the default parameters. The resulting aligned data in bam format was assembled into transcripts using HTSeq (version 0.6.1p2).

\section{RNA-Seq data analyses}

To increase the degree of freedom and power of statistical analysis, additional human skin fibroblast samples from age matched Trisomy 21 and normal unrelated individuals from ref. ${ }^{31}$. Samples GM04616, AG06922, GM08447, GM05756, and GM00969 were used in conjunction with our samples to make a final number of 4 DS fibroblast lines and $52 \mathrm{n}$ lines. With biological replicates, this count rises to 8 and 9 , respectively. The data files have been deposited in the Gene Expression Omnibus (GEO) database under the accession number GSE126910. The entire analysis was performed in R. For differential gene expression analysis, EdgeR and Limma ${ }^{32}$ packages from $\mathrm{R} /$ Bioconductor were used. The correlation between biological replicates (since they were from the same patients) was estimated and this inter-subject correlation was fitted into the linear model to avoid an overfit and inflation of p-values (i.e., reducing the false positives). Counts per million (CPM) have been used after TMMnormalization in the texts and figures. Gene Set Enrichment Analysis (GSEA) of gene sets within the expression data was carried out using mroast functionality from $\operatorname{ROAST}^{33}$, which assesses the overall magnitude and direction of change of a gene set within a comparison in the whole expression profile. Gene sets were considered to be significantly enriched if the adjusted (Benjamini-Hochberg) $p$-value, i.e., FDR ( $q$-value) was $<0.01$ in pairwise comparisons using 100,000 random rotation. ROAST were used on the mTOR targets list, which was obtained from those genes described to be upregulated upon constitutive hyperactivation of mTOR caused by TSC1 $\mathrm{KO} ;{ }^{34}$ the list of transcriptional factors described to be essential for supporting mitophagy activation and the list of transcriptional factors regulating mitochondrial biogenesis are described in Supplementary Table 1.

\section{Preparation of CDNA and qPCR}

cDNA was prepared from total RNA using TaqMan Reverse Transcription Reagent kit N808-0234 (ThermoFisher/Life Technologies) according to manufacturer's instructions and using Random hexamers. Following reverse transcription, 20 ng sample cDNA were loaded in triplicate into wells of a 96-well optical reaction plate containing appropriate target gene primer (see below; Taq-Man validated primers purchased from Life Technologies/ThermoFisher) in a total reaction volume of
$20 \mu$ l. $\beta$-ACTIN (ACTB) was used as a housekeeping gene. qPCR was performed in the ABI Prism 7900HT Sequence Detection System (Applied Biosystems Branchburg, NJ) as described previously ${ }^{14}$. We note here that although PARK2 (PARKIN) levels were negligible via RNA-Seq, qPCR was sufficient to detect and quantify as described in Results. Following qPCR, the target genes were normalized against the housekeeping gene. Results were calculated using the $\Delta \Delta \mathrm{Ct}$ method (Applied Biosystems, Branchburg, NJ Bulletin \#2). Sample values were recalculated and expressed as percent control. Outliers were recognized as values falling beyond two standard deviations of the mean and were discarded from the subsequent analyses.

List of primers used:

Hs01060665_g1ACTB

Hs00197348_m1ATG7

Hs00157205_m1CTSD

Hs01054576_m1FOXO1

Hs01038325_m1PARK2

Hs00260868_m1PINK1

Hs01061917_g1SQSTM1

Hs00177504_m1ULK1

\section{Immunofluorescence}

Fibroblasts were seeded into a 12-well plate with coverslip and grown to $40-60 \%$ confluence before treatment. Following incubation cells were washed with PBS and fixed with $4 \%$ paraformaldehyde at room temperature for $15 \mathrm{~min}$. After permeabilization with $100 \mu \mathrm{g} / \mathrm{mL}$ Digitonin (Sigma D141) in PBS for $15 \mathrm{~min}$, cells were incubated overnight at $4{ }^{\circ} \mathrm{C}$ with LC3 (\#M152-3, MBL for ICC) and with TOM20 (\#sc-11415, Santa Cruz Biotechnology) in PBS with $3 \%$ normal goat serum (v:v). Cells were then washed in PBS and incubated for $1 \mathrm{~h}$ with fluorescent conjugated secondary antibodies (ThermoFisher/Life Technologies). Mitochondrial ROS production was evaluated using MitoSOXTM ( $3 \mu \mathrm{M}$, Molecular Probes) and mitophagy was evaluated using a combination of LysoTracker Red DND-99 (100 nM; L-7528) with Mitotracker Green FM (100 nM; M-7514) according to the manufacturer's instructions (ThermoFisher). Immunofluorescent images for LC3 and TOM20, for MitoSox staining or double labeling with Mitotracker Green and Lysotracker Red were collected on a confocal microscope (Zeiss LSM510). Co-localization and quantification of LC3 puncta per cell were determined using the JACoP Plug-In on Image (NIH) software. Fluorescent images were adjusted for brightness, contrast and color balance using Adobe Photoshop CS (San Jose, CA, USA).

\section{Mitochondrial mass}

Cells were seeded in 96 microplate F-bottom (Greiner Bio-One International \#655090) and incubated with 
MitoTracker Green FM as described above. Fluorescence was measured at $488 \mathrm{~nm}$ using a SpectraMax M5 plate reader (Molecular Devices).

\section{SDS-PAGE and Western blotting}

Following treatment cultures were rinsed with ice-cold


$1 \%$ Triton $\mathrm{X}-100,150 \mathrm{mM} \mathrm{NaCl}, 0.25 \%$ sodium deoxycholate, $0.1 \%$ SDS, $1 \mathrm{mM}$ EDTA, $5 \mathrm{mM} \mathrm{MgCl}_{2}$ ) containing protease inhibitor cocktail (Sigma, St. Louis, MO, USA) and phosphatase inhibitors $(20 \mathrm{mM} \mathrm{NaF}, 10 \mathrm{mM}$ $\beta$-glycerophosphate, $1 \mathrm{mM} \mathrm{Na} \mathrm{VO}_{4}$ ) via sonication and incubation in ice for $30 \mathrm{~min}$. A clear supernatant was obtained by centrifugation of lysates at $15000 \mathrm{rpm}$ for $15 \mathrm{~min}$. Mitochondrial/cytosolic fractionation was performed as described previously ${ }^{35}$. Cells were harvested in hypotonic buffer $\left(15 \mathrm{mM} \mathrm{MgCl}_{2}, 10 \mathrm{mM} \mathrm{KCl}, 10 \mathrm{mM}\right.$ Tris-MOPS, pH 7.4), supplemented with protease inhibitor cocktail and incubated for $15 \mathrm{~min}$ in ice. 1 Volume of $2 x$ Mitobuffer ( $400 \mathrm{mM}$ sucrose, $10 \mathrm{mM}$ TES, $\mathrm{pH}$ 7.2, $100 \mathrm{mM}$ EGTA, $2 \mu \mathrm{M}$ DTT) was added and the cell suspension was homogenized for 40 strokes with a Dounce homogenizer. Samples were centrifuged twice at $900 \times g$ for $10 \mathrm{~min}$ at $4{ }^{\circ} \mathrm{C}$ to eliminate cell nuclei and unbroken cells. The resulting supernatant was centrifuged at $12,000 \times g$ for $15 \mathrm{~min}$ at $4{ }^{\circ} \mathrm{C}$ to recover the heavymembrane pellet enriched in mitochondria, and the resulting supernatant was stored as cytosolic fraction. Protein concentration was determined using the BCA protein assay (Thermo Scientific \#23225); and samples subjected to SDS-polyacrylamide gel electrophoresis followed by Western blotting described previously ${ }^{14}$. Following processing, nitrocellulose membranes (Pall) were subjected to enhanced chemiluminescent substrates (Invitrogen, WP20005 or Millipore WBKL50500) and exposed to X-ray film (Ewen-Parker, RX-B); bands were quantified using Image $(\mathrm{NIH})$ software. Target proteins were normalized against $\beta$-ACTIN or VDAC1, unless otherwise noted.

Antibodies for Western analyses: AKT (\#4685), phospho473-AKT (\#4060), phospho308-AKT (\#2965), ATG3 (\#3415), ATG7 (\#2631), COXIV (\#4850), FOXO1 (\#2880), H3 (\#4499), mTOR (\#2983), NDP52 (\#60732) phospho2448-mTOR (\#2971), P70S6K (\#2708), phospho389-P70S6K (\#9234), TFEB (\#4240), ULK-1 (\#4773), phospho758-ULK1 (\#6888) and VDAC1 (\#4866) from Cell Signaling; p62/SQSTM1 (610832) from MBL; LC3B (NB1002220) and PINK1 (BC100-494) from Novus Biologics; PARKIN (Ab77924) and OPTINEURIN (Ab23666) from Abcam; TOM20 (\#sc-11415) from Santa Cruz Biotechnology; BIII-TUBULIN (SAB4700544) from Sigma.

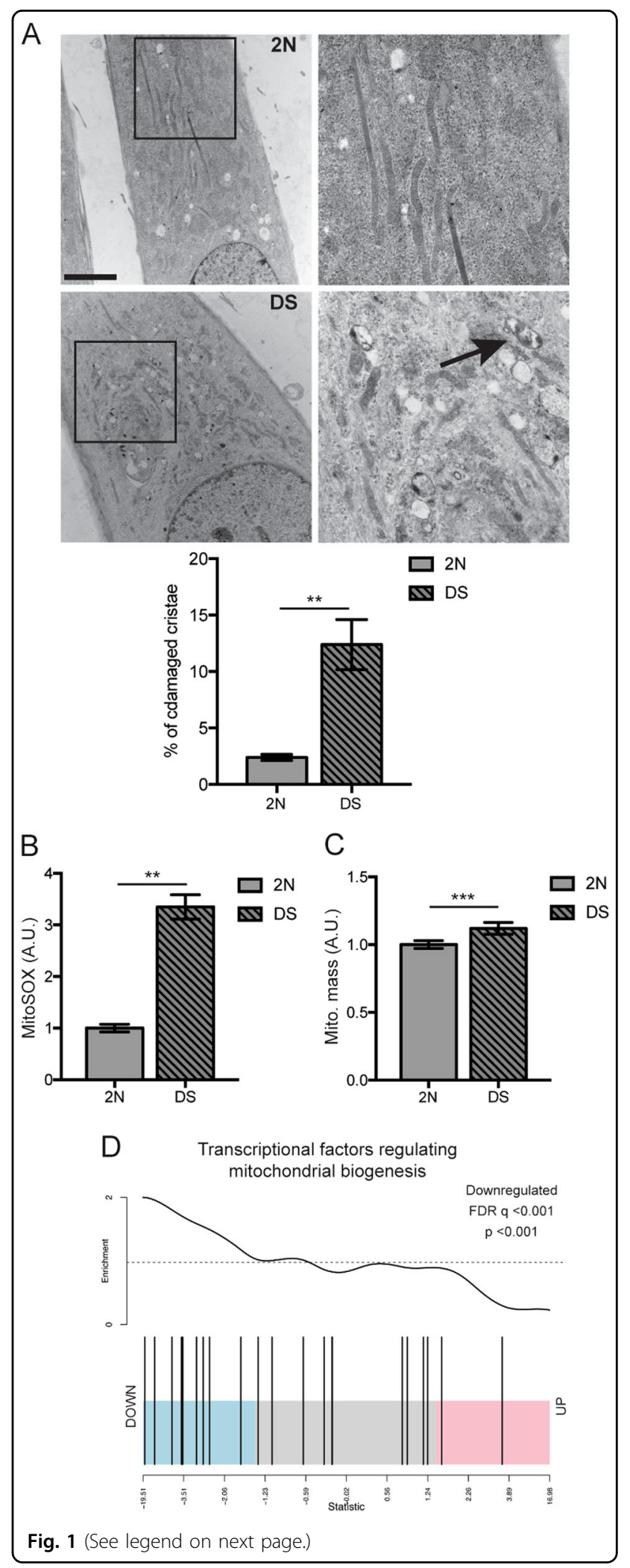


(see figure on previous page)

Fig. 1 Damaged mitochondria accumulation in DS fibroblasts and resulting increase of oxidative stress. a Electron microscopy analysis of $2 \mathrm{~N}$ and DS cells; section indicated by the square is shown at higher magnification in the right panels. Black arrow points to example of an abnormal mitochondrion. Quantification shown as percentage of damaged mitochondria in respect to total number. Scale bar $500 \mathrm{~nm}$. b Quantification of MitoSOX ${ }^{\mathrm{TM}}$ intensity fluorescence reported as fluorescence arbitrary units (A.U.) for three independent experiments. The analysis was done using the software ImageJ. c Quantification of mitochondrial mass using Mitotracker green intensity fluorescence, acquired through SpectraMax M5 Microplate Reader and reported as fluorescence arbitrary units (A.U.) from at least three independent experiments. $\mathbf{d}$ Gene set analysis barcode plot of transcriptomic changes of transcriptional factors (TFs) regulating mitochondrial biogenesis ${ }^{7,42,43}$ (for list of genes, see Supp. Table 1) in DS fibroblasts compared to $2 \mathrm{~N}$ fibroblasts. The vertical bars represent TFs (genes) and are ranked horizontally by moderated t-statistics (x-axis). Pink, blue and grey shaded rectangles mark upregulated, downregulated and genes that are unchanged, respectively. The black trace above the bars shows relative enrichment of genes using Limma's ROAST function (see Methods section). The analysis shows significant downregulation of TFs regulating mitochondrial biogenesis in DS compared to $2 \mathrm{~N}$ fibroblasts. $(p<$ 0.001 ; FDR $<0.001$.. Statistical analysis in $A, B$ and $C$ was performed using Student's $T$-test. $\left({ }^{*} p<0.05 ;{ }^{* *} p<0.01 ;{ }^{* *} p<0.001\right)$

\section{Carbonylation protein assay}

We used the Oxidized Protein Western blot Kit (\#ab178020) from Abcam, as per manufacturer's instructions.

\section{Electron microscopy}

After washing with serum-free medium, cells were fixed with $4 \%$ paraformaldehyde/ $1 \%$ glutaraldehyde/ $5 \%$ sucrose in $0.1 \mathrm{M}$ sodium cacodylate buffer, $\mathrm{pH} 7.2$ (Electron Microscopy Sciences, PA) for $24 \mathrm{~h}$ at RT. Cells then washed three times with $0.1 \mathrm{M}$ sodium cacodylate buffer, post-fixed in $1 \%$ osmium tetroxide and progressively dehydrated in a graded series of ethanols (50-100\%) followed by embedding in Epon (Electron Microscopy Sciences) for at least 3 days at RT. Epon-embedded blocks were sectioned and placed on copper grids for structural analysis via Transmission Electron Microscopy (TEM) with magnification $\times 5800, \times 13500$ or $\times 25000$. EM images in each condition were randomly selected and captured, and the number of damaged mitochondria was manually counted. Vacuoles within mitochondria were quantified upon AZD8055 treatment after 2 and $8 \mathrm{~h}$ and expressed as a ratio of autophagosomes to autolysosomes inside mitochondria/total number of autophagosomes and autolysosomes per field.

\section{Results}

\section{Damaged mitochondria accumulate in DS fibroblasts in} association with oxidative stress

To dissect molecular mechanisms contributing to the DS phenotype, we evaluated alterations to the mitochondrial network. We first examined DS and $2 \mathrm{~N}$ control fibroblasts by TEM and fluorescent imaging techniques to highlight differences in mitochondrial ultrastructure and function. EM analysis revealed significantly more mitochondria with abnormal morphology in DS fibroblasts compared to $2 \mathrm{~N}(p<0.05$; Fig. 1a). Altered cristae organization, such as internal structures collapsed against one side (Fig. 1a) were associated with decreased mitochondrial respiration efficiency and increased reactive oxidative stress (ROS) ${ }^{36}$. Accordingly, DS cells exhibited elevated levels of mitochondrial superoxide ( $p<0.01$; Fig. 1b, Suppl. Fig. 1a), and oxidative carbonylated proteins (Suppl. Fig. 1b) correlating with increased ROS production, confirming earlier studies ${ }^{7-9,37}$. Accordingly, levels of SOD2, the main scavenger of superoxide into the mitochondria ${ }^{38}$, were lowered in DS cells $(p<0.01$; Suppl. Fig. 1c, d), while VDAC1, a critical regulator of mitochondrial metabolic and energetic functions $^{39}$, was unaltered (Suppl. Fig. 1c, d). Transcriptomic analyses comparing gene expression related to mitochondrial function and ROS revealed reduced SOD2 expression (Suppl. Fig. 1e) and down-regulation of other important antioxidant enzymes, including TXNRD1/2, (Suppl. Fig. 1e). However mitochondrial fission and fusion genes regulating mitochondrial dynamics ${ }^{40}$ were unchanged (Suppl. Fig. 1g).

Mitochondrial mass was significantly elevated in DS cells compared to $2 \mathrm{~N}(p<0.001)$ (Fig. 1c). To support this finding, we performed GSEA $^{41}$ using a gene set of relevant transcriptional factors (TFs) regulating mitochondrial biogenesis, including PPARGC1A (PGC-1 $\alpha)$, a master regulator of mitochondrial biogenesis ${ }^{7}, P P A R G$ (PPARgamma), TFAM, STAT3, TFB1M and FOXO1 $1^{42,43}$ showing these were significantly downregulated in DS $(p<$ 0.001 , FDR $q<0.001$; Fig. 1d and Suppl. Fig. 1f). Hence, despite transcriptional evidence for reduced mitochondrial biogenesis, DS cells exhibit increased mitochondrial mass, an accumulation of damaged mitochondria, and an upsurge of ROS, collectively suggesting that clearance of defective mitochondria may be impaired.

\section{Impaired mitophagy responses are associated with PINK1/ PARKIN dysregulation}

To investigate the basis for abnormal accumulation of damaged mitochondria in DS fibroblasts, we assessed the competence of PINK1-PARKIN dependent mitophagy ${ }^{15}$. Notably basal levels of PARKIN protein were $\approx 60 \%$ lower in DS fibroblasts than in $2 \mathrm{~N}$ cells $(p<0.001$, Fig. $2 \mathrm{a}, \mathrm{b})$, concomitant with lowered PARKIN mRNA $(\approx 45 \%, p<$ 0.01; Fig. 2c). By contrast, PINK1 protein and mRNA levels in DS cells were unchanged (Fig. 2a-c).

We next investigated whether the lowered expression of PARKIN could impair mitophagy. Immunoblot analyses of enriched mitochondrial fractions from DS cells show 


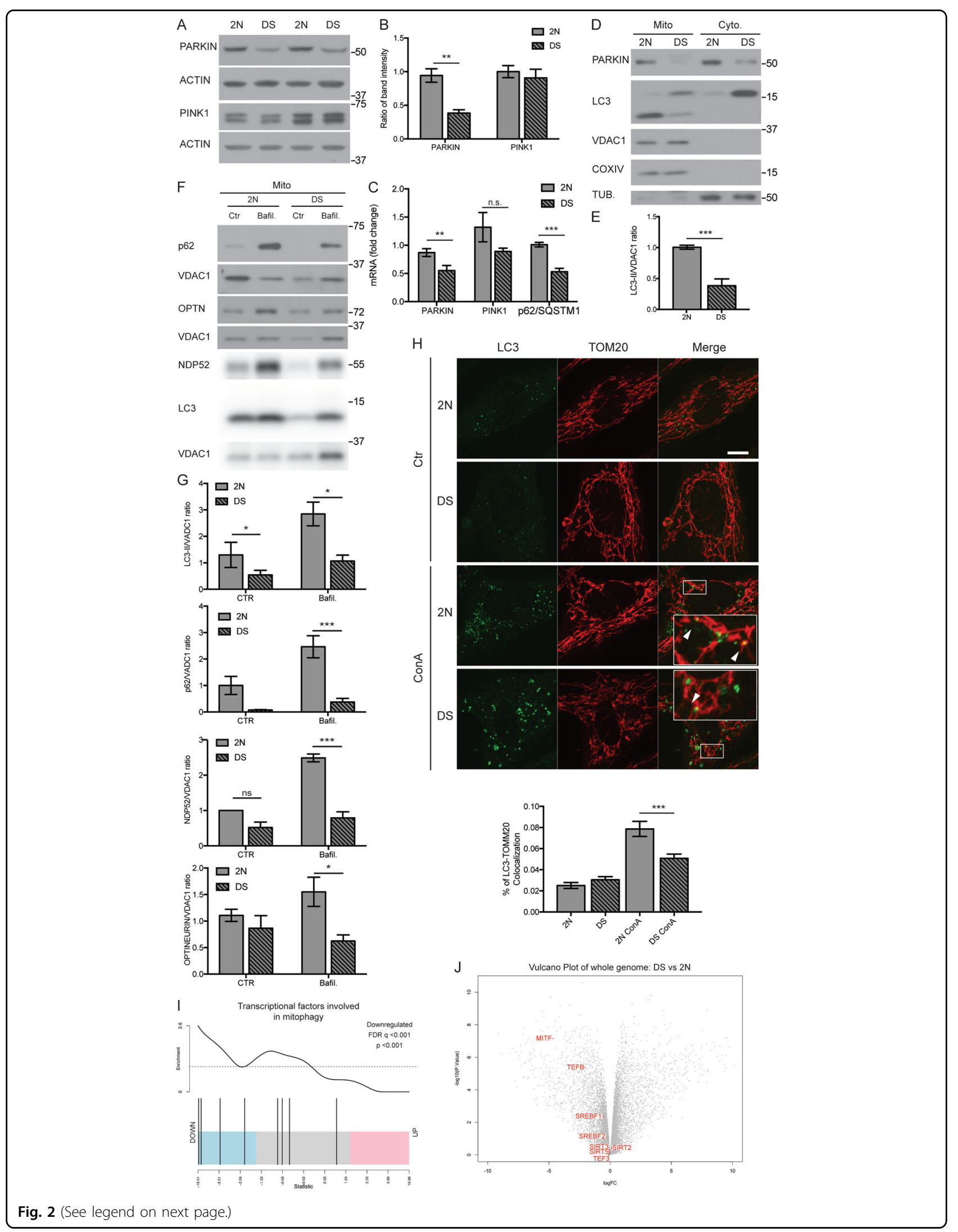


(see figure on previous page)

Fig. 2 PARKIN down regulation and impairment of mitophagy in DS fibroblasts. $\mathbf{a}$, $\mathbf{b}$ Whole-cell extracts from $2 \mathrm{~N}$ and DS cells were analyzed by western blot for PARKIN and PINK1. Each quantitative data was normalized with ACTIN. $(n \geq 3)$. c $2 \mathrm{~N}$ and DS cells were assessed for PARKIN and PINK1 mRNA by quantitative real-time PCR. mRNA levels were normalized to ACTIN mRNA, used as internal control. Data display the fold-changes of PARKIN, PINK1 and SQSTM1 (p62) mRNAs relative to control cells $(n \geq 3)$. d Mitochondrion/cytosol fractionation was performed to assay the presence of PARKIN and LC3-II within the mitochondrial fraction. VDAC1 and COXIV were used as markers of purity for mitochondrial fraction. $\beta$ III-TUBULIN was used as a marker of the cytosolic fraction. e Quantitative data of LC3-II in the mitochondrial fraction, normalized with VDAC1 $(n=5)$.

f Mitochondrial fractionation was performed to assess the accumulation of p62, LC3-II, NDP52 and OPTINEURIN (OPTN) within the mitochondria in untreated and treated fibroblasts with a lysosomal-inhibiting agent, Bafilomycin A (Bafil., $10 \mathrm{nM}$ ) for $6 \mathrm{~h}$. Each protein was normalized with VDAC1 with quantitation in $(\mathbf{g})$. $(n \geq 3) \mathbf{h} 2 \mathrm{~N}$ and DS cells were treated with Concanamycin A (ConA, $50 \mathrm{nM}$ ) for $2 \mathrm{~h}$ to block lysosome function. The cells were fixed and immunostained for LC3 and TOM20 in order to evaluate mitophagy. White arrows point to the co-localization of puncta into the high magnification image of the boxed area. Scale bar $10 \mu \mathrm{m}$. The graph shows the percentage of LC3/TOM20 colocalization calculated by JACoP plugin of ImageJ. Quantitation based on a minimum of 50 cells per conditions from three independent experiments. $\mathbf{i}$ GSEA analysis of transcriptomic changes comparing DS fibroblasts to $2 \mathrm{~N}$ fibroblasts, using a list of transcriptional factors that have been described to be involved in mitophagy ${ }^{46,47}$ (for list of genes, see Supp. Table 1). As described in Fig. 1d, the vertical bars represent genes ranked horizontally by moderated $t$-statistics; Pink, blue and grey shaded rectangles upregulated, downregulated and genes with no change respectively. The plot shows significant downregulation of TFs involved in mitophagy in DS compared to $2 \mathrm{~N}$ fibroblasts $(p<0.001$; FDR $<0.001)$. $\mathbf{j}$ Volcano plots for whole transcriptomes of differentially expressed genes between DS and $2 \mathrm{~N}$ fibroblasts, with TFs involved in mitophagy, used in (I) for GSEA analysis highlighted with labels. Statistical analysis was performed using Student's T-test or one-way ANOVA with Tukey's multiple comparisons test. $\left({ }^{* *} p<0.01 ;{ }^{* *} p<0.001\right)$. Immunoblots reported are from one representative experiment

PARKIN loss was associated with a $60 \%$ decrease in LC3II levels $(p<0.001$; Fig. 2d, e), suggesting a mitophagy deficit compared to $2 \mathrm{~N}$. To exclude elevated degradation of damaged mitochondria as an explanation, we repeated the analyses in the presence of Bafil, a specific inhibitor of lysosomal acidification ${ }^{28}$. Treatment reduced mitochondrial-LC3-II co-localization in DS cells, together with p62, NDP52 and OPTINEURIN, three cargo receptors central for autophagic clearance of mitochondria ${ }^{44}$, supporting the conclusion that initial stages of mitophagy were impaired (Fig. 2f, g). By RNA-seq analysis, no changes were observed in CALCOCO2 (NDP52) and OPTN (OPTINEURIN) mRNA levels, while MAP1LC3B (LC3B) significantly increased between DS and $2 \mathrm{~N}$ cells (Suppl. Fig. 1h). SQSTM1 (p62) showed a trend of decrease that became significant when analyzed by qPCR $(p<0.001$; Fig. 2c).

To further establish a defect in mitophagy, we assessed colocalization between endogenous LC3 puncta and TOM20, a mitochondrial outer membrane protein (Fig. 2h). Consistent with impaired mitophagy initiation, colocalization decreased in DS cells $(p<0.001$; Fig. $2 \mathrm{~h})$ in the presence of the v-ATPase inhibitor, ConA ${ }^{29}$. Notably, basal colocalization was not significantly lowered in DS cells compared to $2 \mathrm{~N}$ cells (Fig. 2h), pointing to lysosomal activity impairment noted previously ${ }^{45}$.

We next evaluated alterations of genes encoding key proteins regulating the mitophagy pathway. GSEA and transcriptomic analysis revealed overall significant downregulation of TFs essential for supporting mitophagy activation $^{46,47}$ in DS (Fig. 2i-j), strengthening links between mitophagy impairment and accumulation of damaged mitochondria.

\section{Alteration of the PINK1/PARKIN pathway is associated with mitophagy deficits}

To investigate the role of decreased PARKIN in impairing mitophagy, we activated mitophagy by treating DS and $2 \mathrm{~N}$ control fibroblasts cells with CCCP ${ }^{15}$. LC3-II levels were elevated in the mitochondrial fraction in response, albeit significantly lower in DS compared to $2 \mathrm{~N}$ $(p<0.05$; Fig. 3a, b), suggesting impaired mitophagy. WB analysis confirmed this observation wherein total lysates showed 70\% lower PINK1 accumulation in DS cells $(p<$ 0.05 vs control; Fig. 3d, f). Indeed, upon mitochondrial membrane depolarization, PINK1 is stabilized on the outer membrane where it promotes PARKIN-mediated mitophagy initiation ${ }^{15}$. In human fibroblasts, p62 seems not to be involved in CCCP-induced mitophagy, as also described in HeLa cells ${ }^{17,48}$. Accordingly, p62 changes were minimal in response to CCCP $(6 \mathrm{~h})$ in $2 \mathrm{~N}$ and DS cells, which nevertheless maintain a modest association with mitochondria irrespective of treatment (Fig. 3a, c).

We then evaluated the delivery of mitochondria to lysosomes by measuring colocalization of Mitotracker green and Lysotracker red. Colocalization of these two markers at baseline and upon CCCP treatment in DS cells $(p<0.001$; Fig. $3 \mathrm{~g}, \mathrm{~h})$ is consistent with a reduced capacity to eliminate damaged mitochondria compared to $2 \mathrm{~N}$. We identified mitochondrial stress induced by CCCP promoting expression of PARKIN and PINK1 genes ${ }^{49,50}$. Herein PARKIN expression via $\mathrm{qPCR}$ analysis was similarly upregulated in both cell lines after $6 \mathrm{~h}$ of CCCP treatment (Fig. 3i); however, we did not see any rise of PARKIN protein levels in DS cells, but significant lowering in $2 \mathrm{~N}$ (Fig. 3d, e), as noted also by Rakovic and coauthors in healthy human fibroblasts ${ }^{18}$. PINK1 


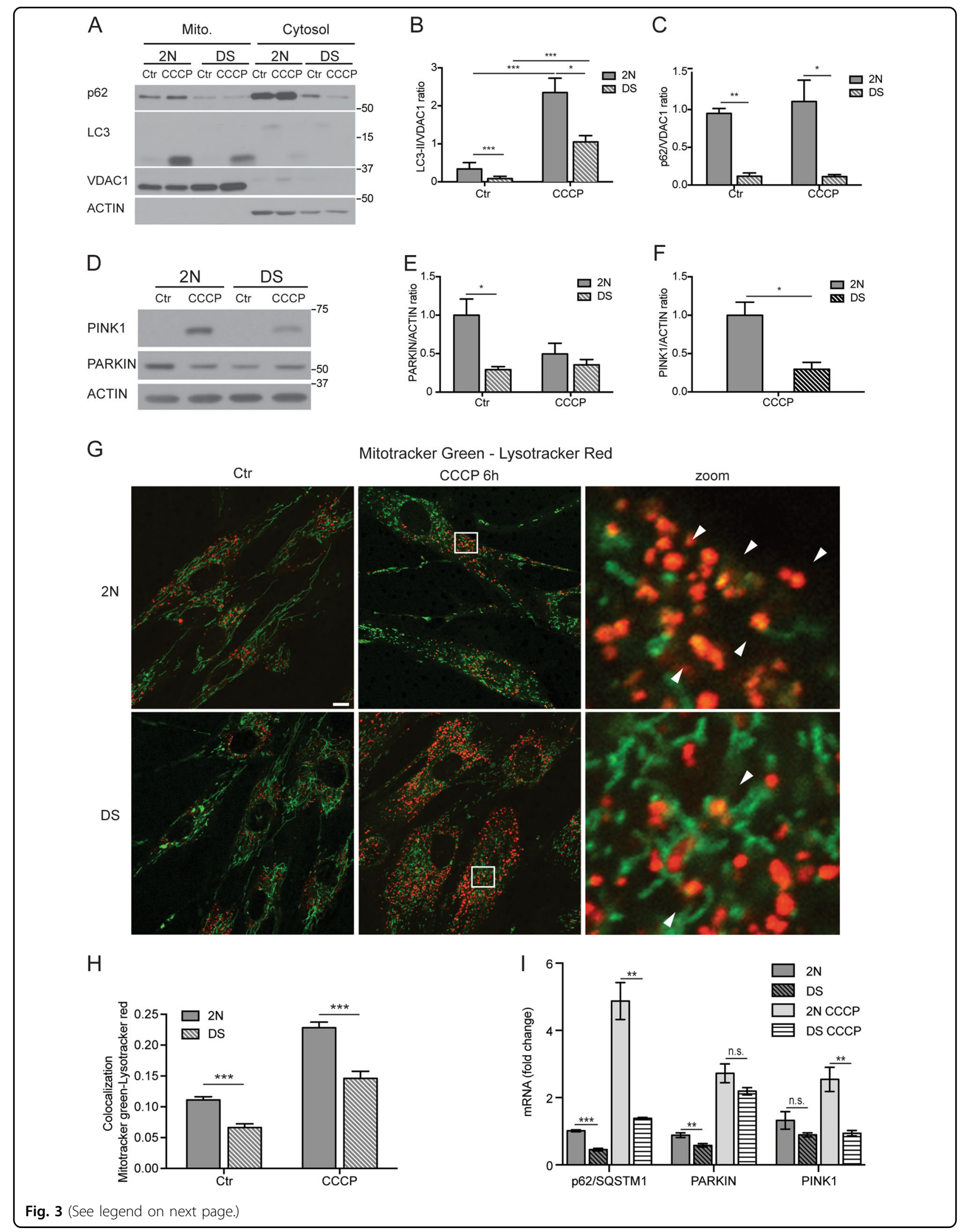


(see figure on previous page)

Fig. 3 Alteration of PARKIN leads to an impairment of mitophagy upon CCCP treatment. a $2 \mathrm{~N}$ and DS were treated with CCCP ( $20 \mu \mathrm{M})$ for $6 \mathrm{~h}$ and mitochondrion/cytosol fractionation was performed to assay the accumulation of LC3-II and p62 within the mitochondrial fraction. VDAC1 and ACTIN were used as a loading control for mitochondrial and cytosolic fractions, respectively. b, c Quantitative data of LC3-II and p62 in the mitochondrial fraction were normalized with VDAC1 $(n=3)$. d The cells were treated with CCCP $(20 \mu \mathrm{M})$ for $6 \mathrm{~h}$ and the whole-cell extracts from $2 \mathrm{~N}$ and DS were analyzed by western blot for PARKIN and PINK1. e Quantification of PARKIN normalized with ACTIN $(n \geq 3)$. d Quantification of PINK1 after $6 \mathrm{~h}$ of CCCP, each quantitative data was normalized with ACTIN $(n \geq 3)$. g Representative confocal images of cells stained with Mitotracker green (mitochondrial marker) and Lysotracker red (lysosomal marker) upon CCCP treatment ( $20 \mu \mathrm{M}$ for $6 \mathrm{~h}$ ). White arrow heads point to the co-localization within puncta from the boxed region at high magnification shown on the right. Scale bar $10 \mu \mathrm{m}$. $\mathbf{h}$ The graph shows the percentage of Mitotracker green /Lysotracker red colocalization calculated by JACoP plugin of ImageJ. Minimum 50 cells per condition were counted from three independent experiments. i $2 \mathrm{~N}$ and DS cells were treated with CCCP $(20 \mu \mathrm{M})$ for $6 \mathrm{~h}$ and were assessed for SQSTM1 (p62), PARKIN and PINK1 mRNA by quantitative real-time PCR. mRNA levels were normalized to ACTIN mRNA, used as internal control. Data display the fold-changes of SQSTM1, PARKIN and PINK1 mRNAs relative to control cells $(n=3)$. Statistical analysis was performed using Student's T-test or one-way ANOVA with Tukey's multiple comparisons test. ( ${ }^{*} p<0.05 ;{ }^{* *} p<0.01 ;{ }^{* * *} p<0.001 ;$ n.s. no significant difference). Immunoblots reported are from one representative experiment

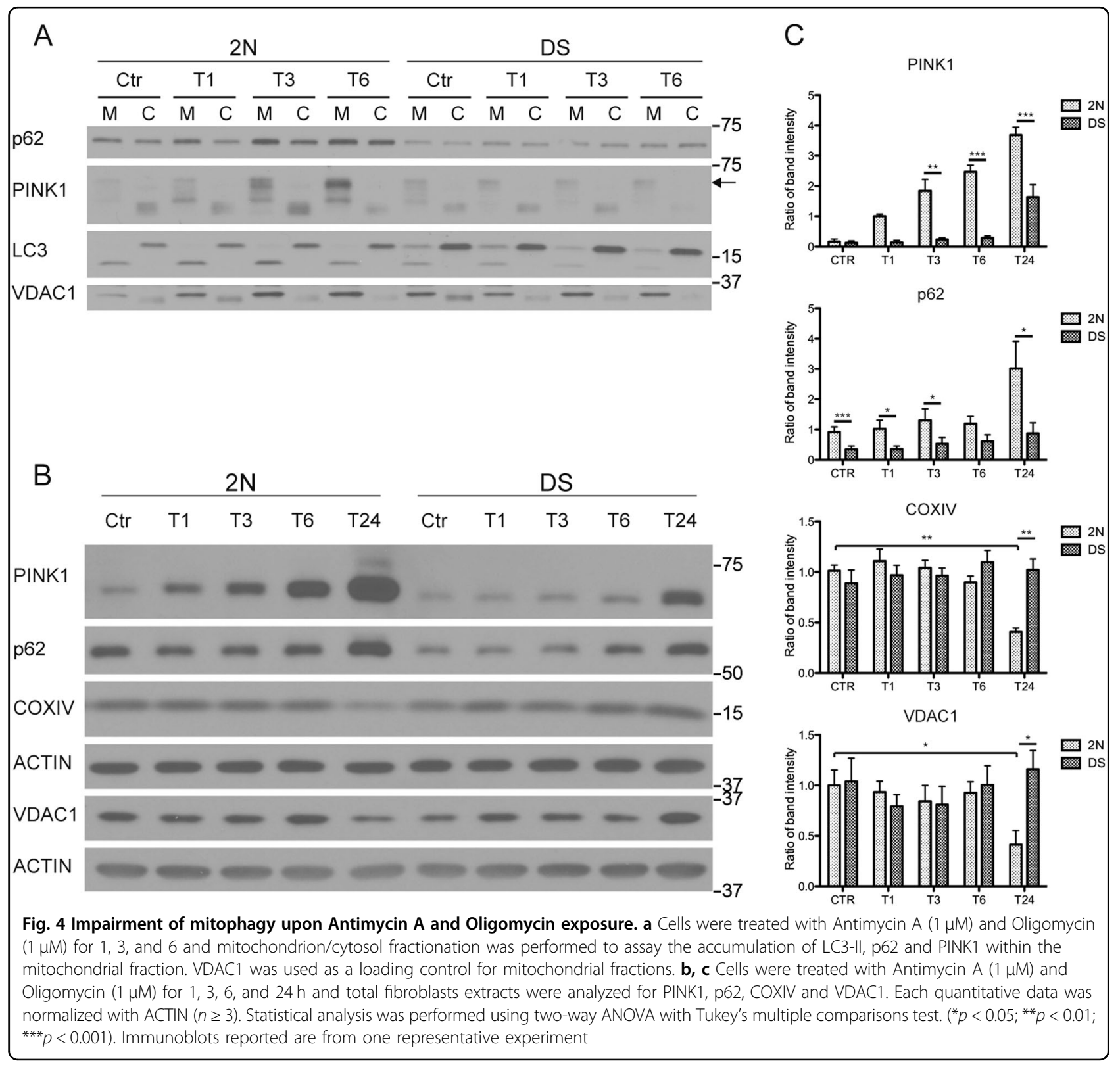




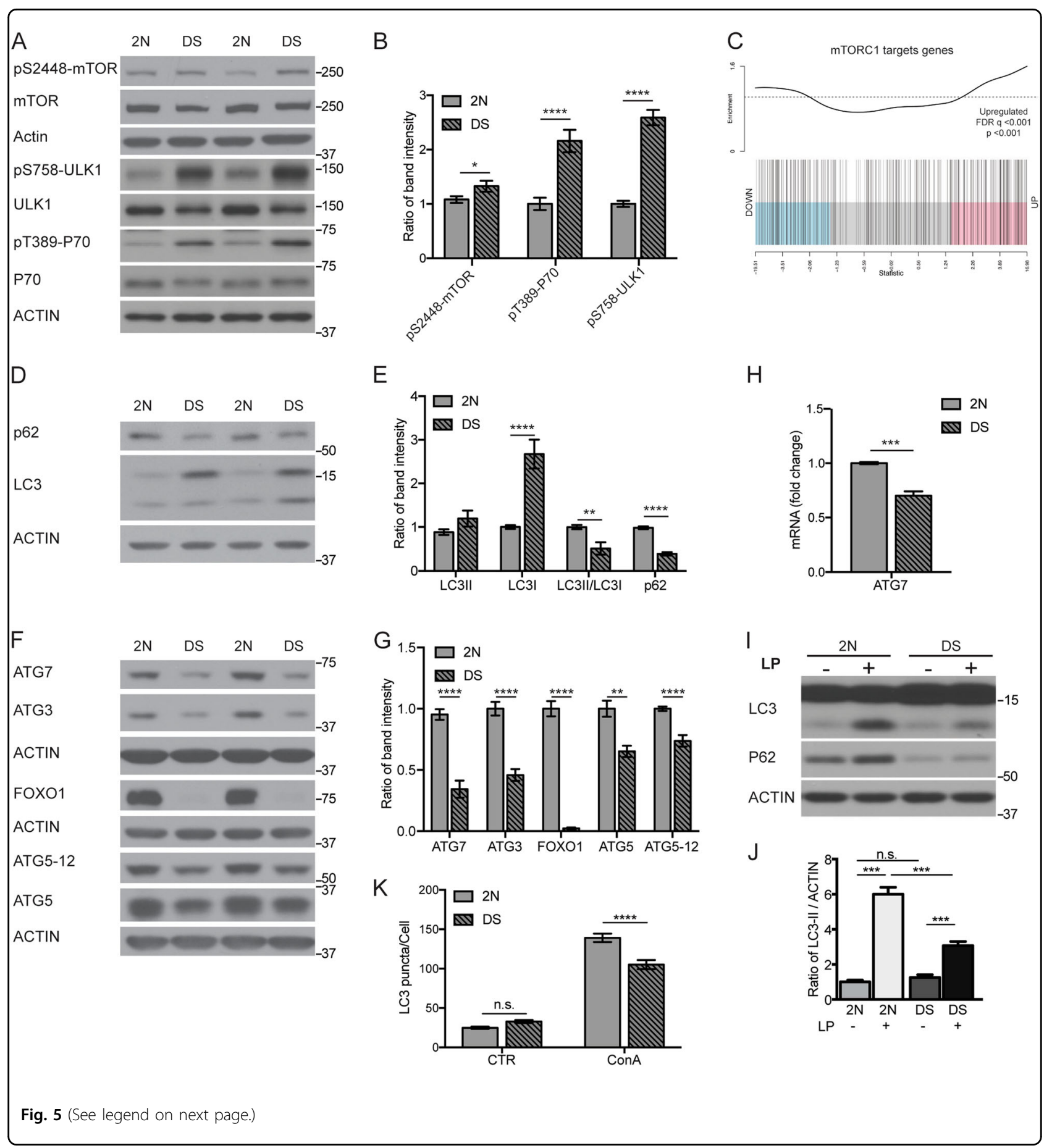

expression was not altered at basal levels (Fig. 3i) and unlike $2 \mathrm{~N}$ cells $(p<0.01$; Fig. $3 \mathrm{i})$, was not efficiently upregulated after CCCP exposure in DS. Although SQSTM1 expression was significantly increased in both cell lines, the rise was greater in $2 \mathrm{~N}$ cells (Fig. $3 \mathrm{i}$ ).

CCCP is commonly used to induce mitophagy, but it can also cause ER stress ${ }^{49}$, and prevent lysosomal acidification $^{51}$. Therefore, we confirmed mitophagy impairment in DS fibroblasts with a milder mitophagy stimulus consisting of a combination of antimycin $\mathrm{A}$ and oligomycin $(\mathrm{AA} / \mathrm{OA})^{28}$. We monitored AA/OA-induced translocation of p62, LC3-II and PINK1 stabilization in enriched mitochondrial fractions, observing a timedependent accumulation of PINK1 and p62 within $1 \mathrm{~h}$ in $2 \mathrm{~N}$ but not in DS cells (Fig. 4a) where neither PINK1 nor p62 significantly changed over $6 \mathrm{hr}$ (Fig. 4a). Of note, 
(see figure on previous page)

Fig. 5 Hyperactivation of mTOR and impairment of autophagy. a Total fibroblasts extracts were subjected to SDS-PAGE and analyzed for mTOR, ULK1 and P70S6K (P70), the phospho-specific antibodies were used to determine mTOR activity. b Phosphorylation levels quantified by densitometry as a ratio of phospho-protein/total protein level ( $n \geq 3)$. c GSEA of transcriptome changes comparing DS fibroblasts to $2 \mathrm{~N}$ fibroblasts, using a list of genes described to be upregulated upon hyperactivated mTOR signaling due to Tsc1 depletion (Shin et al. ${ }^{34}$ ). As described for Figs. $1 \mathrm{~d}$ and $2 \mathrm{i}$, the vertical bars represent genes horizontally ranked by moderated t-statistics; Pink, blue and grey shaded rectangles upregulated, downregulated and genes that are unchanged, and the trace above the bar highlights relative enrichment of genes (see Methods sections for other details). The plot shows significant upregulation of this list of genes in DS compared to $2 \mathrm{~N}$ fibroblasts. $(p<0.01$; FDR $<0.01)$. $\mathbf{d}$ Whole-cell extracts were analyzed by western blot for p62, LC3-1, LC3-II with ACTIN used as a loading control with quantitation in $(\mathbf{e})$. ( $n \geq 3)$. $\mathbf{f}$ Whole-cell extracts were analyzed by western blot for highly relevant autophagic proteins ATG7, ATG3, FOXO1, ATG5 and ATG5-12 complex with ACTIN used as a loading control with quantitation in $(\mathbf{g})$. ( $n \geq 3)$. $\mathbf{h} 2 \mathrm{~N}$ and DS cells were assessed for p62 and AGT7 mRNA by quantitative real-time PCR. mRNA levels were normalized to ACTIN mRNA, used as internal control. Data display the fold-changes of ATG7 mRNAs relative to control cells ( $n \geq 3$, extracts prepared from independent experiments). i Western blot analysis of LC3 and $p 62$ in $2 \mathrm{~N}$ and DS fibroblasts treated with vehicle or both Leupeptin and Pepstatin $A$ ( $L P, 10 \mu M$ for $24 \mathrm{~h}$ ) with quantitation shown in (j). $(n=4)$. $\mathbf{k}$ Quantification of endogenous LC3 puncta structures per cell in the presence or absence of lysosomal inhibitor Concanamycin A (ConA, 50 nM; for $2 \mathrm{~h}$ ) in DS and $2 \mathrm{~N}$ cells. Quantification based upon counts derived from images shown in Fig. $2 \mathrm{~h}$. Quantitation based on a minimum of 70 cells per conditions from three independent experiments. All values are shown as the mean \pm SEM. Statistical analysis was performed using Student's T-test or two-way ANOVA with Tukey's multiple comparisons test. $\left({ }^{*} p<0.05 ;{ }^{* *} p<0.01 ;{ }^{* * *} p<0.001\right.$; n.s. no significant difference). Immunoblots reported are from one representative experiment

AA/OA treatment involves accumulation of p62 in the mitochondrial fraction suggesting mitophagy induced in this manner differs somewhat from CCCP treatment although each revealed a deficit in DS.

Since prolonged mitochondrial depolarization can cause mitochondrial protein depletion due to mitophagymediated removal of damaged mitochondria, we examined total cell extracts. Upon extended AA/OA treatment, PINK1 levels in $2 \mathrm{~N}$ cells rose within $6 \mathrm{~h}$, but the PINK1 response was delayed in DS cells and remained lowered up to $24 \mathrm{~h}(p<0.05$, Fig. $4 \mathrm{~b}, \mathrm{c})$ indicating a deficit in DS cells in PINK1/PARKIN-mediated mitophagy upon mitochondrial damage. Levels of the mitochondrial proteins COXIV and VDAC1 decreased only in $2 \mathrm{~N}$ fibroblasts accompanied by a time-dependent accumulation of PINK1 (Fig. 4b, c). Collectively, these results indicate defective PINK1/PARKIN-mediated mitophagy in DS fibroblasts, further contributing to cellular accumulation of damaged mitochondria.

\section{Macroautophagy induction and autophagosome formation are aberrantly down-regulated in DS fibroblasts}

Mitophagy is a form of selective autophagy but still requires the same protein complexes deployed in macroautophagy to form and clear $\mathrm{APs}^{28}$. We therefore investigated the molecular cascade regulating autophagy flux starting from the AKT-MTOR signaling pathway. Western blot analysis of DS fibroblasts showed that dual phosphorylation on $\mathrm{Thr}^{308} / \mathrm{Ser}^{473}$ of AKT, yielding maximum activity $^{52}$, was significantly increased $(p<0.01$; Suppl. Fig. 2a, b). Next, we investigated whether AKT activation also regulated $\mathrm{mTORC}^{52}$. Consistent with this finding, immunoblot analysis demonstrated increased phosphorylation at $S^{2448}$ within the kinase catalytic domain of mTOR in DS cells, indicating abnormally elevated mTOR activity $(p<0.01$; Fig. $5 \mathrm{a}, \mathrm{b})$ as also reported in DS brain and primary cortical neurons from a DS mouse model ${ }^{25,26,53}$. Consistent with mTOR hyperactivation, phosphorylation of P70 ribosomal S6 kinase (P70S6K), a direct substrate of mTORC1 ${ }^{54}$, was increased $(p<0.001$; Fig. 5a, b). Moreover, increased phosphorylation of ULK1-S ${ }^{758}$, reflected activity inhibition and suppression of autophagy induction in DS fibroblasts relative to $2 \mathrm{~N}$ controls $(p<0.001$; Fig. $5 \mathrm{a}, \mathrm{b})$. Supporting these findings, we interrogated by GSEA analysis genes known to be targeted by hyperactivated mTOR signaling in TSC1 $\mathrm{KO}^{34}$, which revealed an enrichment in DS $(p<0.001$, FDR $q<0.001$; Fig. 5c).

Given that the AKT-mTORC1 cascade negatively regulates autophagy ${ }^{22}$, we investigated whether AP formation was also affected in DS fibroblasts. Despite markedly higher levels of LC3-I protein, correlating with significantly increased MAP1LC3B mRNA levels in DS cells (Suppl. Fig. 1h), we observed a reduction of LC3-II/LC3-I ratio $(-80 \%, p<0.001$; Fig. 5d, e). Accordingly, ATG5 protein, involved with lipidation of LC3 and its family members $^{55}$, was downregulated $(-35 \%, p<0.001)$ (Fig. 5f, g) and in the ATG12-ATG5 conjugated complex (Fig. 5f, g). Moreover, p62 protein $(p<0.001$; Fig. $5 \mathrm{~d}, \mathrm{e})$ and SQSTM1 expression ( $p<0.001$; Fig. $2 \mathrm{c}$ and $3 \mathrm{i})$ were also lowered. Finally, proteins critical for AP elongation, ATG7, a E1-like enzyme, and ATG3, a E2-like enzyme ${ }^{55}$, were markedly downregulated at both the protein $(p<$ 0.001; Fig. 5f, g) and mRNA levels (RNA-seq data, Suppl. Fig. 2c; qPCR for $A T G 7, p<0.001$; Fig. 5h).

Although these observations strongly suggested that autophagy induction was downregulated in DS fibroblasts, we confirmed this directly by measuring AP formation in presence of leupeptin-pepstatin A (LP) to prevent AP clearance. After $24 \mathrm{~h}$ of treatment, LC3-II was lower in DS cells $(p<0.001$; Fig. $5 \mathrm{i}, \mathrm{j})$. As previously shown, impaired lysosomal degradation of autophagic substrates ${ }^{45}$ causes 





(see figure on previous page)

Fig. 6 Rescue of autophagy and mitophagy induction defects in DS by mTOR inhibition. a $2 \mathrm{~N}$ and DS fibroblasts were treated with AZD8055 (AZD; $0.1 \mu \mathrm{M}$ ), an mTOR specific inhibitor, for $2 \mathrm{~h}$ and whole-cell extracts were assessed for p62, LC3, P70S6K (P70) and mTOR; the phospho-specific antibodies were used to determine mTOR activity. b LC3-II levels following AZD treatment quantified and normalized with ACTIN $(n=4)$. $\mathbf{c}$ Electron microscopy analysis of $2 \mathrm{~N}$ and DS cells after $2 \mathrm{~h}$ AZD treatment. Black arrows point to autophagosome/autolysosomes with engulfed mitochondria. Scale bar $500 \mathrm{~nm}$. d Quantification of autophagosome/autolysosomes (autophagic vacuoles, AVs) with engulfed mitochondria after 2 and $8 \mathrm{~h}$ AZD treatment, graph shows the ratio AVs with Mitochondria/AVs total number. e The cells were treated with AZD $(0.1 \mu \mathrm{M})$ for $2 \mathrm{~h}$ and mitochondrion/ cytosol fractionation was performed to assay the accumulation of PARKIN, p62, ATG7 and LC3-II within the mitochondrial fractions. VDAC1 was used as a loading control. $\mathbf{f}, \mathbf{g}$ Western evaluation of PARKIN levels upon $2 \mathrm{~h}$ AZD treatment and relative quantification normalized with ACTIN. $\mathbf{h}$. $2 \mathrm{~N}$ and DS cells were treated with AZD for $2 \mathrm{~h}$ and $8 \mathrm{~h}$ and were assessed for PARKIN and PINK1, ATG7, FOXO1, ULK1, and CTSD (Cathepsin D) mRNA by quantitative real-time PCR. mRNA levels were normalized to ACTIN mRNA, used as internal control. Data display the fold-changes of gene expression relative to control cells $(n \geq 3)$. Statistical analysis was performed using one-way or two-way ANOVA with Tukey's multiple comparisons test for Real Time PCR. Immunoblots reported are from one representative experiment. $\left({ }^{*} p<0.05 ;{ }^{* *} p<0.01\right.$; ${ }^{* * *} p<0.001$ )

an abnormally low rate of clearance of LC3-II-positive APs and thus, basal LC3-II levels were not significantly lowered in DS cells compared to $2 \mathrm{~N}$ cells (Fig. 5e, j). Quantification of LC3 puncta number/cell further verified the autophagy flux impairment in DS cells with a marked decrease of LC3 puncta number compared to $2 \mathrm{~N}$ in the presence of ConA $(p<0.0001$; Fig. $5 \mathrm{k})$. Collectively, these data show that AP formation and maturation in DS is downregulated at multiple levels: from depressed induction (increased AKTmTORC1 axis signaling) to lowered expression of key ATG genes required for AP formation shown herein, and likely additional deficits of lysosomal clearance ${ }^{45}$.

\section{Inhibition of mTOR rescues mitophagy defects and macroautophagy suppression in DS fibroblasts}

To further investigate the relationships of altered PINK1/PARKIN dependent mitophagy and downregulated autophagy induction to accumulation of damaged mitochondria, we inhibited mTORC1 activity with AZD8055 (AZD) to promote autophagy and possibly mitophagy. As expected, AZD diminished mTORC1 activity within $2 \mathrm{~h}$ as evidenced by decreased p-P70 and pmTOR (Fig. 6a). At this time point, LC3-II levels rose in both cell lines (Fig. 6a, b) but to a greater extent in DS likely due to impaired lysosomal degradation (Fig. 5e, j), previously established in these cells ${ }^{45}$. To assess AZD effects on mitophagy, we analyzed EM images to detect mitochondria sequestered within APs. APs containing mitochondria were detected in both cell lines (Fig. 6c, arrows), implying that mTOR inhibition promotes mitophagy. The numbers of AP/AL containing mitochondria significantly increased after $2 \mathrm{~h}$ of treatment in $2 \mathrm{~N}$ cells and after $8 \mathrm{~h}$ in DS cells (Fig. 6d). Western blot analysis confirmed this result, showing a rise of mitochondrial LC3-II level and PARKIN translocation in mitochondrial fractions upon $2 \mathrm{~h}$ of AZD (Fig. 6e) in both DS and $2 \mathrm{~N}$ cells. Moreover, at this time point, we detected a significant upregulation of PARKIN total protein levels compared to basal condition in DS cells (Fig. 6f, g).
We evaluated whether mTOR inhibition through AZD could reverse the downregulated expression in DS cells of components involved in AP formation (e.g., ATG7 and FOXO1) and mitophagy (PARKIN) found herein. Reports revealed how mTORC1 negatively regulates autophagy also at the transcriptional level by inhibiting, for example, the members of microphthalmia/transcription factor $\mathrm{E}$ (MiT/TFE) subfamily (such as TFEB and TFE 3$)^{56,57}$. In fact, upon mTOR inhibition (starvation or ATPcompetitive mTOR inhibitors), TFEB and TFE3 translocate into the nucleus to promote expression of target genes supporting the activation of autophagy and lysosomal biogenesis ${ }^{56,57}$. qPCR analysis revealed AZD treatment induced elevation in PINK1, ULK1 and CTSD (Cathepsin D) expression in both cell lines (Fig. 6h) in a time-dependent manner. Intriguingly, in DS cells, AZD significantly upregulated PARKIN and there was a trend toward increases for ATG7 and FOXO1, which were downregulated in untreated DS cells ( $p \leq 0.01$, Fig. 6h). These results demonstrate rescue of both autophagy and PINK1/PARKIN related mitophagy deficits in DS cells when autophagy induction is stimulated.

Finally, we repeated the immunostaining for endogenous LC3 and TOM20 upon AZD treatment with and without ConA (Fig. 7). Following $2 \mathrm{~h}$ treatment, the colocalization of $\mathrm{LC}^{+}$puncta with TOM20 increased significantly over baseline, with further enhancement after ConA $(p \leq 0.001$, Fig. 7b). Notably, the combination treatment suppressed the differences between the two cell lines observed in presence of ConA alone. Collectively, our findings demonstrate that mTOR inhibition, which restores macroautophagy and mitophagy activity in DS cells, rescues the abnormal accumulation of damaged mitochondria.

\section{Discussion}

We used a multi-faceted approach to comprehensively analyze mitophagy and autophagy deficits in DS fibroblasts. While confirming significant accumulation of 


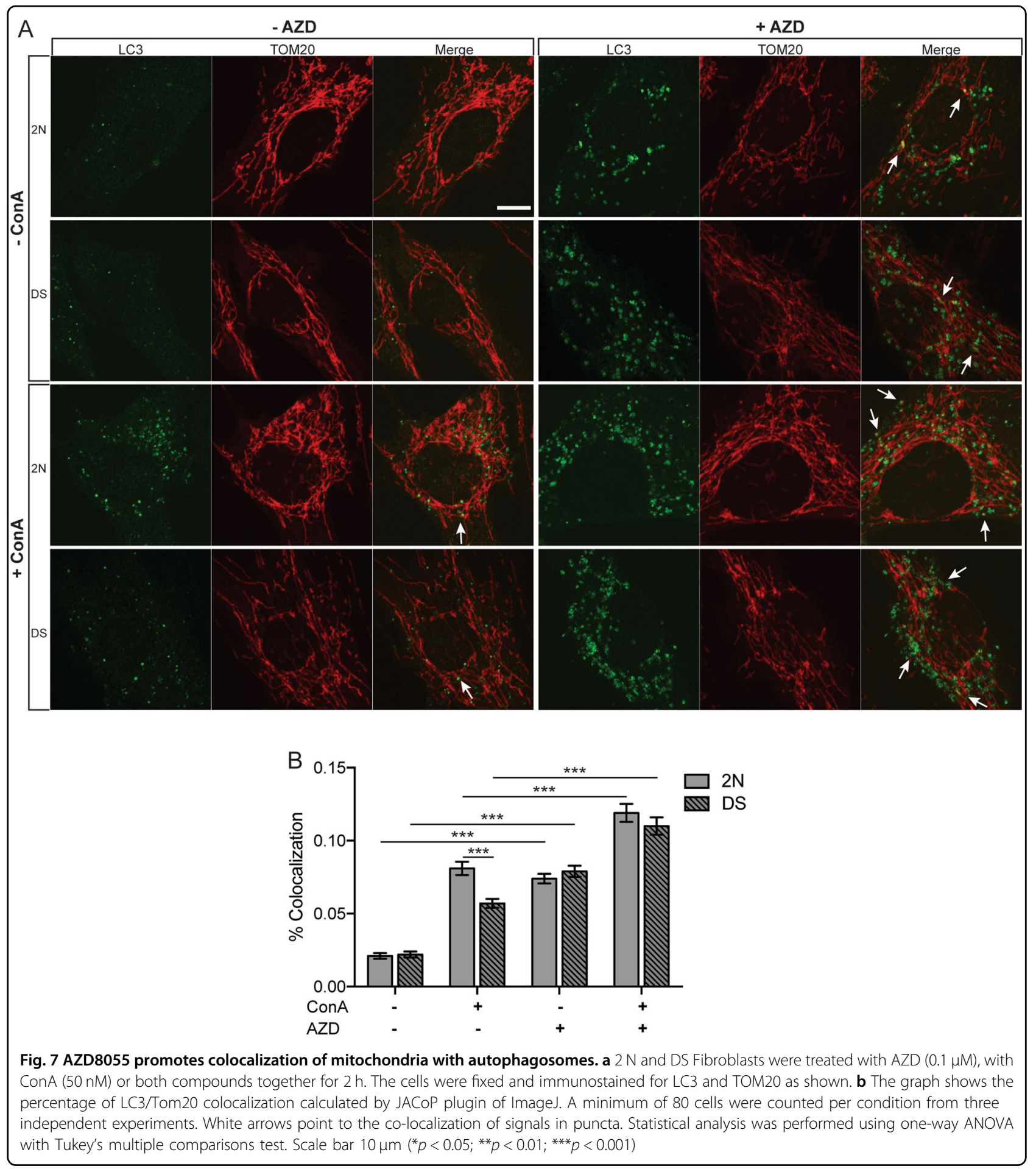

damaged mitochondria associated with increased oxidative stress ${ }^{7-9,37}$, we found that factors involved in transcriptional control of mitochondrial biogenesis and metabolism, including PGC- $1 \alpha^{7}$, were lowered. Despite the possibility that of lowered mitochondrial biogenesis, total mitochondrial mass was increased, raising suspicions that deficient mitophagy could explain increased numbers of damaged mitochondria and total mitochondrial mass. Consistent with this possibility, we established multiple lines of evidence (Fig. 8) revealing two sources of mitophagy suppression in DS cells: firstly, downregulated PARKIN associated with perturbed PINK1-generated 


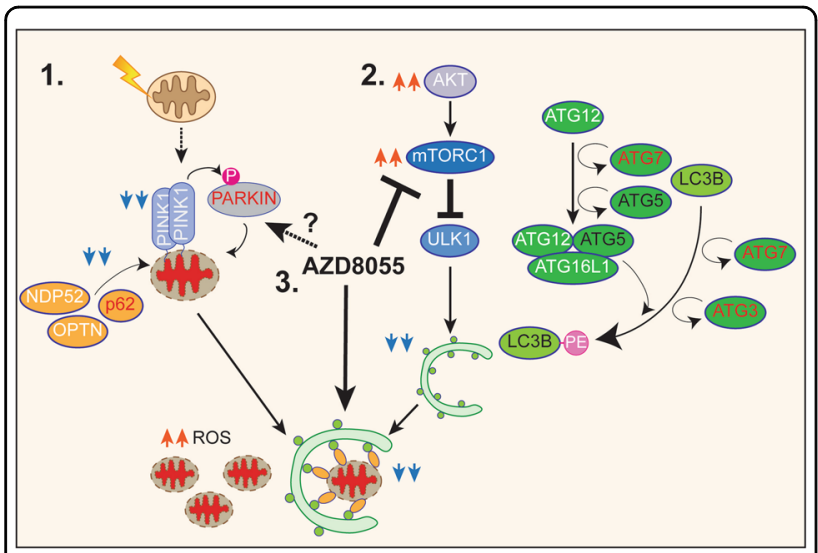

Fig. 8 Proposed model illustrating the autophagy and mitophagy defects in Down Syndrome fibroblasts. Our findings indicate that (a) PARKIN/PINK1-mediated mitophagy pathway is altered in DS human fibroblasts leading to an accumulation of damaged mitochondria and thus to increase of ROS levels. We also find that macroautophagy is negatively regulated at different levels (b) mTOR is hyperactivated leading to an inhibition of autophagy induction and crucial proteins involved in the autophagosome formation, are down-regulated, including ATG7 and FOXO1. c AZD8055 (AZD), an inhibitor of both of mTOR complex 1 (TORC1) and complex 2 (TORC2), rescues mitophagy and autophagy flux, thereby promoting the clearance of damaged mitochondria. The extensive autophagic deficits likely contribute in different ways to the severe symptoms described in DS individuals, leading to the supposition that TORC1-TORC2 inhibition can be a promising therapeutic option for Down Syndrome. Factors downregulated in DS cells highlighted as follows: titles in red denote those downregulated at both the mRNA and protein level, black downregulated at the protein level, white are those that are unchanged. Blue arrowheads indicate those pathways downregulated while orange arrowheads those upregulated. See text for further details

mitophagy signaling and secondly, decreased mitophagy activity from downregulated mTOR-dependent macroautophagy. Notably, both impairments of mitophagy were rescued by blocking $\mathrm{mTOR}$ with AZD, suggesting the therapeutic potential of this class of autophagy enhancers.

Mitophagy is a specialized type of autophagy but uses core machinery similar to non-selective macroautophagy to support sequestration and clearance steps. In fact, activation of the PINK1-PARKIN axis recruits on mitochondria proteins mediating AP biogenesis including ULK1, DFCP1 and WIPI1 ${ }^{17,55}$. ULK1 activation coordinates mitophagy ${ }^{58}$ and is required to further recruit downstream ATG proteins ${ }^{59}$. Accordingly, Ulk1 knockout mice exhibit mitophagy defects and accumulation of aberrant mitochondria ${ }^{60}$. We also found that mTORC1 hyperactivation in DS fibroblasts catalyzed the inactivating phosphorylation of ULK1, resulting in decreased autophagy induction and potentially a decline in mitophagy. The AKT-mTORC1 axis was also shown to be hyperactivated in DS cells, as supported by transcriptomic analysis, which corroborates and extends findings in DS human brain and primary cortical neurons from the DS mouse model Ts1Cje ${ }^{25,26,53}$. Although the basis for the hyperactivated AKT-mTORC1 cascade in DS cells is not yet identified and may be multifactorial, mTORC1 hyperactivation accounts for the strong suppression of autophagy, and as our AZD findings indicate, of mitophagy.

We identified components of two ubiquitin-like conjugation systems critical for AP initiation and maturation $^{28}$ that were downregulated, including ATG7, ATG3, FOXO1 (as direct ATG7 interactor) ${ }^{61}$, ATG5 and ATG512 complex. These findings support down-regulation of autophagy induction at multiple levels and not only from lowered ULK1 activity due to mTORC1-mediated $S^{758}$ phosphorylation. Furthermore, increased oxidative stress in DS cells resulting from diminished elimination of dysfunctional mitochondria, may further compromise the autophagy-mitophagy process, thereby establishing a negative regulatory feedback loop. For example, high ROS generation alters PARKIN function and can disrupt its E3 ligase activity $^{62,63}$ and compromise mitophagy. More recently, Frudd and collaborators have shown that oxidative stress inhibits autophagy by directly oxidizing ATG7 and ATG $3^{64}$, which we also find downregulated in trisomic fibroblasts.

mTORC1 negatively regulates autophagy in multiple ways, including blocking ULK1 activity and preventing nuclear translocation of TFs supporting AP and lysosomal biogenesis ${ }^{54,65,66}$. Importantly, we established that mTOR inhibition by AZD8055 $5^{27}$ both reversed autophagy induction deficits in DS cells within $2 \mathrm{~h}$ of treatment and restored mitophagy levels in DS fibroblasts. Moreover, AZD restored expression of autophagic and mitophagic genes downregulated in untreated DS including ATG7, FOXO1 and PARKIN while significantly elevated PINK1 mRNA levels, thus underscoring its positive effect on mitophagy induction. The beneficial effects of AZD on DS fibroblasts at multiple levels may be related to its inhibitory effect on both mTOR complexes. AZD blocks mTORC2 which negatively regulates AKT and activates FOXO1 and $\mathrm{FOXO}^{27}$ which play critical roles in regulating autophagic gene expression ${ }^{67,68}$ and control PINK1 transcription after growth factor deprivation ${ }^{69}$ or diabetes-induced mitophagy ${ }^{70}$. These observations could explain the positive effects we observed on PINK1 expression upon AZD treatment.

In conclusion, we have extensively characterized multifactorial alterations of autophagy and mitophagy pathways in DS fibroblasts, providing novel insights into the molecular pathogenesis of Down syndrome. The restoration of autophagy and mitophagy functions with AZD treatment reveals common sites of regulation, suggesting promising approaches to ameliorating deficits associated with Down syndrome in various organs, including brain. 


\section{Acknowledgements}

Supported by the National Institute on Aging P01 AG017617 (R.A.N.). M.B. was supported by a "Fondazione Umberto Veronesi" fellowship.

\section{Author details}

${ }^{1}$ Center for Dementia Research, Nathan Kline Institute, Orangeburg, NY, USA. ${ }^{2}$ Department of Psychiatry, New York University Langone Medical Center, New York, NY, USA. ${ }^{3}$ Department of Biology, University of Rome Tor Vergata, Rome, Italy. ${ }^{4}$ Experimental Sciences, Universidad Francisco de Vitoria, Pozuelo de Alarcón, Madrid, Spain. ${ }^{5}$ Department of Cell Biology, New York University Langone Medical Center, New York, NY, USA. ${ }^{6}$ NYU Neuroscience Institute, New York University Langone Medical Center, New York, NY, USA

\section{Conflict of interest}

The authors declare they have no conflict of interest.

\section{Publisher's note}

Springer Nature remains neutral with regard to jurisdictional claims in published maps and institutional affiliations.

Supplementary Information accompanies this paper at (https://doi.org/ 10.1038/s41419-019-1752-5).

Received: 20 March 2019 Revised: 29 May 2019 Accepted: 18 June 2019 Published online: 22 July 2019

\section{References}

1. Hattori, M. The DNA sequence of human chromosome 21. Nature 405 311-319 (2000).

2. Dekker, A. D., De Deyn, P. P. \& Rots, M. G. Epigenetics: The neglected key to minimize learning and memory deficits in Down syndrome. Neurosci. Biobehav. Rev. 45, 72-84 (2014).

3. Hartley, D. et al. Down syndrome and Alzheimer's disease: Common pathways, common goals. Alzheimer's Dement 11, 700-709 (2015).

4. Di Domenico, F. et al. mTOR in Down syndrome: Role in Aß and tau neuropathology and transition to Alzheimer disease-like dementia. Free Radic. Biol. Med. 114, 94-101 (2018).

5. Busciglio, J. et al. Altered metabolism of the amyloid $\beta$ precursor protein is associated with mitochondrial dysfunction in Down's syndrome. Neuron 33 677-688 (2002)

6. Valenti, D., Manente, G. A., Moro, L., Marra, E. \& Vacca, R. A. Deficit of complex I activity in human skin fibroblasts with chromosome 21 trisomy and overproduction of reactive oxygen species by mitochondria: involvement of the cAMP/PKA signalling pathway. Biochem. J. 435, 679-688 (2011).

7. Piccoli, C. et al. Chronic pro-oxidative state and mitochondrial dysfunctions are more pronounced in fibroblasts from down syndrome foeti with congenital heart defects. Hum. Mol. Genet. 22, 1218-1232 (2013).

8. Helguera, P. et al. Adaptive downregulation of mitochondrial function in down syndrome. Cell Metab. 17, 132-140 (2013).

9. Gimeno, A. et al. Decreased cell proliferation and higher oxidative stress in fibroblasts from down syndrome fetuses. Preliminary study. Biochim. Biophys. Acta - Mol. Basis Dis. 1842, 116-125 (2014).

10. Shukkur, E. A. et al. Mitochondrial dysfunction and tau hyperphosphorylation in Ts1Cje, a mouse model for Down syndrome. Hum. Mol. Genet. 15, 2752-2762 (2006)

11. Galluzzi, L. et al. Molecular definitions of autophagy and related processes. EMBO J. 36, 1811-1836 (2017).

12. Boya, P., Reggiori, F. \& Codogno, P. Emerging regulation and functions of autophagy. Nat. Cell Biol. 15, 713-720 (2013).

13. Nixon, Ra The role of autophagy in neurodegenerative disease. Nat. Med. 19 983-997 (2013).

14. Bordi, M. et al. Autophagy flux in CA1 neurons of Alzheimer hippocampus: Increased induction overburdens failing lysosomes to propel neuritic dystrophy. Autophagy 12, 1-17 (2016).

15. Pickrell, A. M. \& Youle, R. J. Review The Roles of PINK1, Parkin, and Mitochondrial Fidelity in Parkinson's Disease. Neuron 85, 257-273 (2015).
16. Koyano, F. et al. Ubiquitin is phosphorylated by PINK1 to activate parkin. Nature (2014). https://doi.org/10.1038/nature13392

17. Lazarou, M. et al. The ubiquitin kinase PINK1 recruits autophagy receptors to induce mitophagy. Nature (2015). https://doi.org/10.1038/nature14893

18. Rakovic, A. et al. Phosphatase and tensin homolog (PTEN)-induced Putative Kinase 1 (PINK1)-dependent ubiquitination of endogenous parkin attenuates mitophagy: Study in human primary fibroblasts and induced pluripotent stem cell-derived neurons. J. Biol. Chem. 288, 2223-2237 (2013).

19. Hosking, R. mTOR: The master regulator. Cell 149, 955-957 (2012).

20. Nazio, F. et al. mTOR inhibits autophagy by controlling ULK1 ubiquitylation, self-association and function through AMBRA1 and TRAF6. Nat. Cell Biol. 15 406-416 (2013).

21. Bar-Peled, L. \& Sabatini, D. M. Regulation of mTORC1 by amino acids. Trends Cell Biol 24, 400-406 (2014).

22. Laplante, M. \& Sabatini, D. M. mTOR signaling in growth control and disease. Cell 149, 274-293 (2012).

23. Feng, Y., He, D., Yao, Z. \& Klionsky, D. J. The machinery of macroautophagy. Cell Res. 24, 24-41 (2014)

24. Yoshii, S. R. \& Mizushima, N. Autophagy machinery in the context of mammalian mitophagy. Biochim. Biophys. Acta Mol. Cell Res. (2015). https://doi.org/ 10.1016/j.bbamcr.2015.01.013

25. Iyer, A. M. et al. mTOR hyperactivation in Down syndrome hippocampus appears early during development. J. Neuropathol Exp. Neurol. 73, 671-683 (2014).

26. Perluigi, M. et al. Neuropathological role of PI3K/Akt/mTOR axis in Down syndrome brain. Biochim. Biophys. Acta Mol. Basis Dis. 1842, 1144-1153 (2014).

27. Chresta, C. M. et al. AZD8055 is a potent, selective, and orally bioavailable ATPcompetitive mammalian target of rapamycin kinase inhibitor with in vitro and in vivo antitumor activity. Cancer Res. 70, 288-298 (2010).

28. Klionsky, D. J. et al. Guidelines for the use and interpretation of assays for monitoring autophagy. Autophagy 12, 1-222 (2016).

29. Lee, J.-H. et al. Presenilin 1 maintains lysosomal Ca2+ homeostasis via TRPML1 by regulating VATPase-mediated lysosome acidification. Cell Rep. 12, 1430-1444 (2015).

30. Benjamin, D., Colombi, M., Moroni, C. \& Hall, M. N. Rapamycin passes the torch a new generation of mTOR inhibitors. Nat. Rev. Drug Discov. 10, 868-880 (2011).

31. Letourneau, A. et al. Domains of genome-wide gene expression dysregulation in Down's syndrome. Nature 508, 345-350 (2014).

32. Ritchie, M. E. et al. limma powers differential expression analyses for RNAsequencing and microarray studies. Nucleic Acids Res. 43, e47 (2015).

33. Wu, D., Lim, E., Vaillant, F., Visvader, J. E. \& Smyth, G. K. ROAST: rotation gene set tests for complex microarray experiments. Bioinformatics 26, 2176-2182 (2010).

34. Shin, J. et al. Characterization of developmental defects in the forebrain resulting from hyperactivated mTOR signaling by integrative analysis of transcriptomic and proteomic data. Sci. Rep. 7, 2826 (2017).

35. De Zio, D. et al. The DNA repair complex Ku70/86 modulates Apaf1 expression upon DNA damage. Cell Death Differ. 18, 516-527 (2011).

36. Cogliati, S., Enriquez, J. A. \& Scorrano, L. Mitochondrial cristae: where beauty meets functionality. Trends Biochem. Sci. 41, 261-273 (2016).

37. Busciglio, J. \& Yankner, B. Apoptosis and increased generation of reactive oxygen species in Down's syndrome neurons in vitro. Nature 378, 776-779 (1995).

38. Flynn, J. M. \& Melovn, S. SOD2 in mitochondrial dysfunction and neurodegeneration. Free Radic. Biol. Med. 62, 4-12 (2013).

39. Shoshan-Barmatz, V., Ben-Hail, D., Admoni, L., Krelin, Y. \& Tripathi, S. S. The mitochondrial voltage-dependent anion channel 1 in tumor cells. Biochim. Biophys. Acta 1848, 2547-2575 (2014).

40. van der Bliek, A., Shen, Q. \& Kawajiri, S. Mechanisms of mitochondrial fission and fusion. Cold Spring Harb Perspect Biol. 5, 1-16 (2013).

41. Subramanian, A. et al. Gene set enrichment analysis: a knowledge-based approach for interpreting genome-wide expression profiles Aravind. Proc. Nat/. Acad. Sci. USA 102, 15545-15550 (2005)

42. Zhu, J., Wang, K. Z. Q. \& Chu, C. T. After the banquet: Mitochondrial biogenesis, mitophagy, and cell survival. Autophagy 9, 1663-1676 (2013).

43. Gleyzer, N., Vercauteren, K. \& Scarpulla, R. C. Control of mitochondrial transcription specificity factors (TFB1M and TFB2M) by nuclear respiratory factors (NRF-1 and NRF-2) and PGC-1 family coactivators. Mol. Cell. Biol. 25, 1354-1366 (2005).

44. Pickles, S., Vigié, P. \& Youle, R. J. Mitophagy and quality control mechanisms in mitochondrial maintenance. Curr. Biol. 28, R170-R185 (2018). 
45. Jiang, Y. et al. Lysosomal dysfunction in Down syndrome is APP-dependent and mediated by APP-BCTF (C99). J. Neurosci. pii: 0578-19 (2019). https://doi. org/10.1523/JNEUROSCI.0578-19.2019.

46. Nezich, C. L., Wang, C., Fogel, A. I. \& Youle, R. J. MiT / TFE transcription factors are activated during mitophagy downstream of Parkin and Atg5. J. Cell Biol 210, 435-450 (2015).

47. Ivatt, R. M. et al. Genome-wide RNAi screen identifies the Parkinson disease GWAS risk locus SREBF1 as a regulator of mitophagy. Proc. Natl Acad. Sci 111 8494-8499 (2014).

48. Wong, Y. C. \& Holzbaur, E. L. F. Optineurin is an autophagy receptor for damaged mitochondria in parkin-mediated mitophagy that is disrupted by an ALS-linked mutation. Proc. Natl Acad. Sci. 111, E4439-E4448 (2014).

49. Bouman, L. et al. Parkin is transcriptionally regulated by ATF4: evidence for an interconnection between mitochondrial stress and ER stress. Cell Death Differ. 18, 769-782 (2011).

50. Gómez-Sánchez, R. et al. Mitochondrial impairment increases FL-PINK1 levels by calcium-dependent gene expression. Neurobiol. Dis. 62, 426-440 (2014).

51. Ashrafi, G. \& Schwarz, T. L. The pathways of mitophagy for quality control and clearance of mitochondria. Cell Death Differ 20, 31-42 (2012).

52. Zoncu, R., Efeyan, A. \& Sabatini, D. M. mTOR: from growth signal integration to cancer, diabetes and ageing. Nat. Rev. Mol. Cell Biol. 12, 21-35 (2011).

53. Troca-Marín, J. A., Alves-Sampaio, A. \& Montesinos, M. L. An increase in basal BDNF provokes hyperactivation of the Akt-mammalian target of rapamycin pathway and deregulation of local dendritic translation in a mouse model of Down's syndrome. J. Neurosci. 31, 9445-9455 (2011).

54. Laplante, M. \& Sabatini, D. M. Regulation of mTORC1 and its impact on gene expression at a glance. J. Cell Sci. 126, 1713-1719 (2013).

55. Lamb, Ca, Yoshimori, T. \& Tooze, Sa The autophagosome: origins unknown biogenesis complex. Nat. Rev. Mol. Cell Biol. 14, 759-774 (2013).

56. Settembre, C. et al. TFEB links autophagy to lysosomal biogenesis. Science $\mathbf{3 3 2}$, 1429-1433 (2011).

57. Martina, Ja, Diab, H. I., Li, H. \& Puertollano, R. Novel roles for the MiTF/TFE family of transcription factors in organelle biogenesis, nutrient sensing, and energy homeostasis. Cell. Mol. Life Sci. 71, 2483-2497 (2014).
58. Wu, W. et al. ULK1 translocates to mitochondria and phosphorylates FUNDC1 to regulate mitophagy. EMBO Rep. 15, 566-575 (2014).

59. Itakura, E., Kishi-Itakura, C., Koyama-Honda, I. \& Mizushima, N. Structures containing Atg9A and the ULK1 complex independently target depolarized mitochondria at initial stages of Parkin-mediated mitophagy. J. Cell Sci. 125 1488-1499 (2012)

60. Egan, D. F. et al. Phosphorylation of ULK1 (hATG1) by AMP-activated protein kinase connects energy sensing to mitophagy. Science 331, 456-461 (2011).

61. Zhao, Y. et al. Cytosolic FoxO1 is essential for the induction of autophagy and tumour suppressor activity. Nat. Cell Biol. 12, 665-675 (2010).

62. Meng, F. et al. Oxidation of the cysteine-rich regions of parkin perturbs its E3 ligase activity and contributes to protein aggregation. Mol. Neurodegener. 6, 34 (2011).

63. LaVoie, M. J., Cortese, G. P. Ostaszewski, B. L. \& Schlossmacher, M. G. The effects of oxidative stress on parkin and other E3 ligases. J. Neurochem. 103, 2354-2368 (2007).

64. Frudd, K., Burgoyne, T. \& Burgoyne, J. R. Oxidation of Atg3 and Atg7 mediates inhibition of autophagy. Nat. Commun. 9, 95 (2018).

65. Settembre, C. et al. A lysosome-to-nucleus signalling mechanism senses and regulates the lysosome via mTOR and TFEB. EMBO J. 31, 1095-1108 (2012).

66. Shimobayashi, M. \& Hall, M. N. Making new contacts: the mTOR network in metabolism and signalling crosstalk. Nat. Rev. Mol. Cell Biol. 15, 155-162 (2014).

67. Wang, $Y$, Zhou, $Y$. \& Graves, D. T. FOXO transcription factors: Their clinical significance and regulation. Biomed Res. Int. 13 (2014). https://doi.org/10.1155/ 2014/925350.

68. Milan, G. et al. Regulation of autophagy and the ubiquitin-proteasome system by the FoxO transcriptional network during muscle atrophy. Nat. Commun. $\mathbf{6}$ 6670 (2015).

69. Mei, Y. et al. FOXO3a-dependent regulation of Pink1 (Park6) mediates survival signaling in response to cytokine deprivation. Proc. Natl Acad. Sci. 106, 5153-5158 (2009)

70. Li, W. et al. FoxO1 promotes mitophagy in the podocytes of diabetic male mice via the PINK1/parkin pathway. Endocrinology 158, 2155-2167 (2017). 